\title{
Brain Tumor Detection Using Gray Level Co-Occurrence Matrix Feature Extraction Technique
}

\author{
SheelaS ${ }^{\mathrm{a}, 1}$, R.Prema $^{\mathrm{b}}, \mathrm{S}$ Ramya $^{\mathrm{b}}$, B.Thirumahal $^{\mathrm{b}}$ \\ ${ }^{a}$ Assistant Professor, ' Department of ECE, Rajalakshmi Institute of Technology, \\ Chennai, India \\ ${ }^{b}$ Department of ECE, Rajalakshmi Institute of Technology, Chennai, India
}

\begin{abstract}
From each and every passing year, the world has always witnessed a rise in the number of cases of brain tumor. Brain tumor classification and detection is that the most critical and strenuous task within the field of medical image processing while human aided manual detection leads to imperfect divination and diagnosing. Brain tumors have high heterogeneity in appearance and there is a same feature between tumor and non-tumor tissues and thus the extraction of tumor regions from MRI scan images becomes unyielding. A Gray Level Cooccurrence Matrix( GLCM) is applied on MRI scan images to detect tumor and non-tumor regions in brain. The main aim of medical imaging is to extract meaningful information accurately from the images. The method of detecting brain tumor from an MRI scan images are often classified into four categories: PreProcessing, Skull Stripping, Segmentation and have Feature Extraction.
\end{abstract}

Keywords: Brain Tumor, GLCM, Segmentation, Feature Extraction, Classification

\section{Introduction}

A brain tumor is an unusual cell in the brain. Your cranium, which encases your brain, is exceptionally reserved. Any development interior such a confined space can cause issues. Brain tumors can be classified as a Malignant tumors (Cancerous) and Benign tumors(Non-Cancerous) [1]. When kind or dangerous tumors develop, they can cause the pressure interior your cranium to extend. This may cause brain harm, and it can be life-threatening. Brain tumors are categorized as essential or auxiliary. An essential brain tumor begins in your brain. Numerous essential brain tumors are kind. An auxiliary brain tumor, too known as a metastatic brain tumor, happens when cancer cells spread from another organ such as lung or breast to brain. A brain tumor called an intracranial tumor, is an anomalous mass of tissue in which cells develop and increase wildly, apparently unchecked by the components that control typical cells. More than 150 distinctive brain tumors have been reported, incorporate tumors that begin from the tissues of the brain or the brain's quick environment. Essential tumors are

\footnotetext{
${ }^{1}$ Sheela.S, Assistant Professor, Rajalakshmi Institute of Technology,Chennai,India. Email id:ssheela97@gmail.com
} 
characterised as glial or non-glial (created on or within the structures of the brain, counting nerves, blood vessels and organs) and generous or malignant. Secondary Brain Tumors incorporate tumors that emerge some place within the body (such as breast or lungs) and move to the brain, ordinarily through the circulation system. Metastatic or Auxiliary Brain Tumors are considered cancer and are threatening. Preprocessing is a first step to improve the picture quality and remove the noise. Skull Stripping is the process that removes the skull layer of the brain image. Segmentation is an important task in the field of medical image analysis and for radiological evaluation or computer aided diagnosis system, classification plays major role [2]. The final step is the classification to classify the brain image whether it is a benign or malignant. Praveen et.al [3] proposed a technique using Fuzzy C-Means (FCM) and SVM classifier for brain tumor detection. In this, first the MRI images are enhanced. Then, skull skipping is done using morphological operations. Finally, from the segmented image GLCM features are extracted to classify the suspicious region using SVM classifier. Several research works are carrier out to detect the brain tumor. H. S. Abdulbaqi et.al [4] developed an algorithm using Markov Random Field technique to classify brain tumor from MRI images. In this technique, input MRI images are converted into 2D images and segmentation is done using thresholding technique. Mind Tumor Location Utilizing Self Versatile K-Means Grouping is developed by Navpreet Kaur [5].

\section{Methodology:}

This paper creates a novel calculation to identify the sort of brain tumor called lower review glioma by expanding the fundamental novel bolster vector machine. At first the Brain tumor pictures are recovered from the database and they are pre-processed to remove the commotion display within the foundation. Furthermore, the highlights of the irregular and ordinary brain pictures are extracted. Thirdly the double step SVM is executed. Within the to begin with step of double SVM, the SVM show is prepared to classify the images utilizing the highlights extricated from the anomalous and ordinary brain pictures. Within the moment step, the SVM demonstrate is prepared to classify lower review glioma and other distinctive sort. Lower review glioma is the starting tumor organize and it can be distinguished with higher precision utilizing the over said calculation which can certainly offer assistance the specialists to pre plan the surgery some time recently animosity.

\subsection{Image processing}

An image pre-processing point to move forward the picture data by smothering the undesired twists and enhances some of the picture highlights that will be supportive in further handling. The objective of Pre processing is to get rid of the commotion and to supply Differentiate Upgrade to improve the picture quality. 


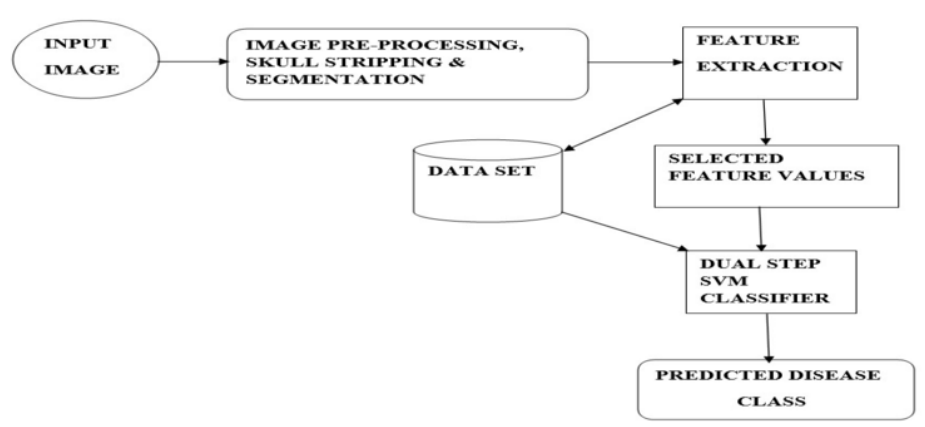

Figure 1. Block diagram of proposed model

\subsection{Grayscale Imaging:}

Grayscale may be a run of update gray without clear color. The darkest possible shade is dark, which is that the complete absence of transmitted or reflected light. The lightest possible shade is white, the entire transmission or reflection of daylight in the least unmistakable wavelengths. So due to the above reasons to begin with we change over our MRI picture to be pre-processed in grayscale picture.

\subsection{Median Filter}

This is the first common method which used for noise disposal. It may be a 'non-linear' filtering method. Typically want to eliminate 'Salt and Pepper noise' from the greyscale image. Middle channel is predicated on the average esteem of pixels. The preferences of median channel are proficient in decreasing Salt and Pepper noise and Dot noise. Moreover, the edges and boundaries are protected. The main disadvantages are complexity and time consumption as compared to cruel filter.

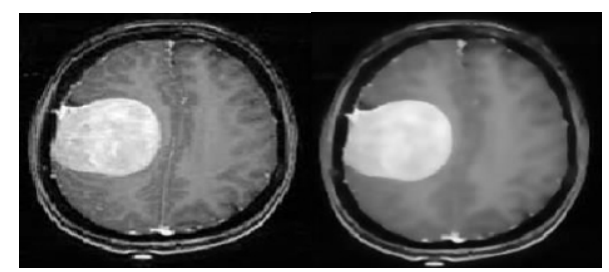

Figure 2. a.Input image b.Median filtered image

\subsection{Skull Stripping}

Skull removal could be a vital step in medical image process as a result of the background of the magnetic resonance imaging image not containing any helpful data, and it solely will increase the time interval. To removed the Otsu's portion from the magnetic resonance imaging pictures in 3 steps. These 3 steps are: a) Otsu Thresholding: For Otsu's removal, initially we tend to used Otsu's Thresholding technique that mechanically calculates the brink price and segments the image into background and foreground. during this technique, the brink that's designated minimizes the intra-class variance, outlined as a weighted total of deviations of the 2 
categories. b) Connected part Analysis: At the last stage of our Otsu's removal step, we tend to used connected part analysis to extract solely the brain region and as a consequence the Otsu's half was removed.

\subsection{Segmentation}

Segmentation may be a troublesome step in medical imaging since the pictures are as well complex. In segmenting handle, the picture is part into several partitions steady with their color, concentrated etc. This makes a difference to observe and analyze the little print of the pictures and supply exact comes about. There are several segmentation strategies.

\subsection{Threshold Image}

Threshold division is one of the simplest segmentation strategies. The strategy is based on threshold esteem which can convert the grayscale image is one of the best division strategies. The method is based on limit esteem which can convert the grayscale picture into double organize. In the threshold division, there are a few methods where we utilize neighbourhood strategies which adjust the edge value on each pixel to the nearby picture characteristics for division.
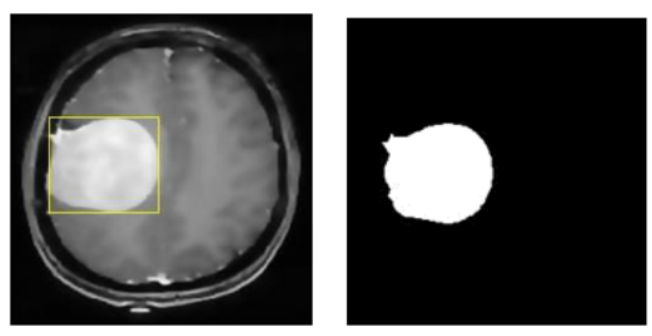

Figure 3. Image segmented using Thresholding based algorithm

\subsection{Feature Extraction}

Classification is done using texture based features and statistical based features. Among several features, energy, correlation, mean, standard deviation, centroid is extracted from the segmented image.

\section{Gray-level Co-occurrence Matrix}

Co-Occurrence matrix is generated and then, texture features are measured from the Co-Occurrence matrix. GLCM is used in several applications like biomedical, remote sensing techniques.etc. Basic of GLCM surface considers the connection between two neighbouring pixels in one balanced, as the moment arrange surface. The gray esteem connections in a target are changed into the co-occurrence network space by a given bit veil such as 33, 55, 77 and so forward. Within the change from the picture space into the co-occurrence lattice space, the neighbouring pixels in one or a few of the eight characterized bearings can be utilized; regularly, four course such as $0^{\circ}, 45^{\circ}, 90^{\circ}$, and 
$135^{\circ}$ is at first respected, and its switch heading (negative heading) can be moreover numbered under consideration.

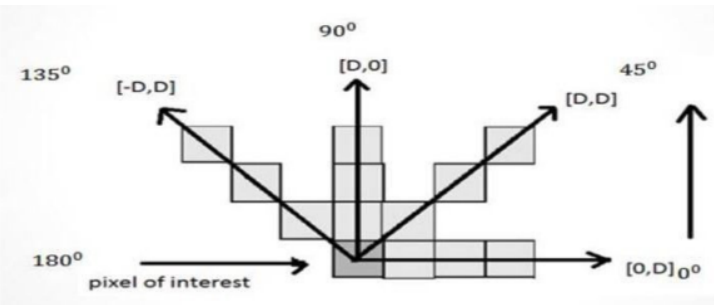

Figure 4. GLCM Directions

\subsection{Classification}

Image classification incorporates classification of pictures into sub categories. It is considered a really critical and troublesome assignment. The classifier procedure is required to classify the data set into categories.

\section{Support Vector Machine(SVM)}

One of the supervised machine learning model is Support Vector Machine (SVM) which uses algorithms for classification. It offers tall precision and performs speedier forecast when compared to Naive Bayes calculation. It can be utilized in both classification and relapse issues. It out-performs with tall dimensional space and with a clear edge of separation.

Here we are utilizing Double Step SVM classification in arrange to classify ordinary as well as irregular and Lower- grade Vs Higher-grade. In arrange to classify climate, the tumor is typical or anomalous we go for SVM1 classifier which employments GLCM Highlights. And, in arrange to classify Lower-grade or Highergrade glioma we go for SVM2 classifier which employments Locale Property Features.

\section{Conclusion}

This extend, different procedures that are being utilized to detect the brain tumor from MRI scan images of brain are evaluated. The proposed strategy has the capability to create compelling comes about indeed in case of tall density of the tumor. The proposed venture will identify the presence of brain tumor with $99 \%$ accuracy.

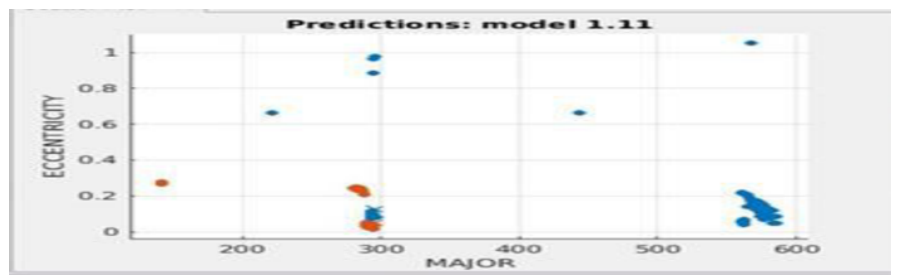

Figure 5. Output of SVM Classifier 


\section{References}

[1] Mohan G, Subashini MM. MRI based medical image analysis: Survey on brain tumor grade classification. Biomed Signal Process Control 2018;39:139-61.

[2] Dhawan, A. P., A Review on Biomedical Image Processing and Future Trends, Computer Methods and Programs in Biomedicine, Vol. 31, No.3-4, 1990, pp.141-183.

[3] Parveen, \& Singh, Amritpal. (2015). Detection of brain tumor in MRI images, using combination of fuzzy c -means and SVM. 98102. 10.1109/SPIN.2015.7095308.

[4] H. S. Abdulbaqi, MohdZubir Mat, A. F. Omar, I. S. B. Mustafa and L. K. Abood, Detecting brain tumor in Magnetic Resonance Images using Hidden Markov Random Fields and Threshold techniques, 2014 IEEE Stud ent Conference on Research and Development, Batu Ferringhi, 2014, pp. 1-5.

[5] Navpreet Kaur and Manvinder Sharma. Brain tumor detection using self-adaptive Kmeans clustering. 2017 International Conference on Energy, Communication, Data Analytics and Soft Computing (ICECDS) (2017): 1861- 1865.

[6] Ambeth Kumar.V.D et.al., .Performance Improvement Using an Automation System for Segmentation of Multiple Parametric Features Based on Human Footprint.for the Journal of Electrical Engineering \& Technology . vol. 10, no. 4, pp.1815-1821 , 2015. [http://dx.doi.org/10.5370/JEET.2015.10.4.1815]

[7] Ambeth Kumar.V.D et.al. , A Survey on Face Recognition in Video Surveillance. Lecturer Notes on Computational and Mechanism, Vol. 30, pp: 699-708, 2019

[8] Ambeth Kumar.V.D, Precautionary measures for accidents due to mobile phone using IOT.Clinical eHealth, Volume 1, Issue 1, March 2018, Pages 30-35.

[9] Ambeth Kumar.V.D et,al, Enhancement in Footprint Image using Diverse Filtering Technique. Procedia Engineering journal, Volume 8, No.12, 1072-1080, 2012 . [doi:10.1016/j.proeng.2012.01.965]

[10] Nanagasabapathy.K et.al, Validation system using smartphone luminescence. IEEE International Conference on Intelligent Computing, Instrumentation and Control Technologies (ICICICT), Pages: 235 - 239, 6-7 July 2017, Kannur, India

[11] Aravindh.B et.al, A novel graphical authentication system for secure banking systems.IEEE International Conference on Smart Technologies and Management for Computing, Communication, Controls, Energy and Materials, Pages: 177 - 183, 2-4 Aug. 2017, Vel Tech University, Chennai, India

[12] Ruphitha.S.V et.al, .Management of Major Postpartum Haemorrhage by using Zigbee protocol - A Review. 2021 6th International Conference on Inventive Computation Technologies (ICICT) (DOI: 10.1109/ICICT50816.2021.9358757 )

[13] Indhumathi.M et.al . Healthcare Management of Major Cardiovascular Disease-A review. 2021 6th International Conference on Inventive Computation Technologies (ICICT), (DOI: 10.1109/ICICT50816.2021.9358519) 